\title{
Persistência na água e influência de herbicidas utilizados na lavoura arrozeira sobre a comunidade zooplanctônica de Cladocera, Copepoda e Rotifera
}

\author{
Water persistence and influence of herbicides utilized in rice paddy about zooplankton \\ community of Cladocers Copepods and Rotifers
}

\author{
Geovane Boschmann Reimche ${ }^{\mathrm{I}}$ Sérgio Luiz de Oliveira Machado ${ }^{\mathrm{II}}$ \\ Jaqueline Ineu Golombieski ${ }^{\mathrm{III}}$ Joele Schmitt Baumart ${ }^{\mathrm{IV}}$ Neiva Braun ${ }^{\mathrm{V}}$ \\ Enio Marchesan ${ }^{\mathrm{VI}}$ Renato Zanella ${ }^{\mathrm{VII}}$
}

\section{RESUMO}

Em lavoura de arroz irrigado, é utilizada uma grande quantidade de agroquímicos que, dependendo da sua persistência a campo e toxicidade, podem contaminar corpos d'água e afetar organismos vivos. Com o objetivo de determinar o efeito de concentrações de campo dos herbicidas Clomazone, Quinclorac, Metsulfuron-methyl e Propanil na comunidade zooplanctônica (Cladocera, Copepoda e Rotifera), conduziuse um experimento em viveiros de aqüicultura, de março a

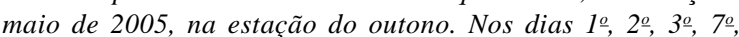
$10^{\circ}, 18^{\circ}, 31^{\circ}$ e $51^{\circ}$ após a aplicação dos herbicidas, foram coletadas amostras de água para se determinarem parâmetros físico-químicos da água, concentração dos herbicidas e comunidade zooplanctônica. Os parâmetros médios da qualidade da água foram: oxigênio dissolvido (3,5mg $\left.\mathrm{L}^{-1}\right)$, temperatura $\left(20,1^{\circ} \mathrm{C}\right), \mathrm{pH}(6,0)$, dureza total $\left(18 \mathrm{mg} \mathrm{L}^{-1}\right.$ de $\left.\mathrm{CaCO}_{3}\right)$ e alcalinidade total ( $9 \mathrm{mg} \mathrm{L}^{-1}$ de $\mathrm{CaCO}_{3}$ ). A ordem decrescente de persistência dos herbicidas na água foi Clomazone = Quinclorac > Propanil > Metsulfuron-methyl, com média de $31,31,10$ e 7 dias, respectivamente. Os resultados indicaram que os herbicidas provocaram poucas alterações na densidade de organismos dos grupos Rotifera e Copepoda (Adulto $e$ Nauplio). A densidade do grupo Cladocera permaneceu baixa para todo o período experimental.

Palavras-chave: agroquímicos, Crustacea, organismos nãoalvos, qualidade de água, Sul do Brasil, viveiros de aqüicultura.

\section{ABSTRACT}

In the rice paddy field it is used a large amount of agrochemical that, depending on their field persistence and toxicity, can contaminate water bodies and may affect living organism. With the objective of determining the effect of field concentrations of Clomazone, Quinclorac, Metsulfuron-methyl and Propanil herbicides on zooplankton community (Cladocers, Copepods and Rotifers), it was carried an experiment in aquaculture ponds, during March to May 2005, in autumn season. In the $1^{\text {st }}, 2^{\text {nd }}, 3^{\text {rd }}, 7^{\text {th }}, 10^{\text {th }}, 18^{\text {th }}, 31^{\text {th }}$ and $51^{\text {th }}$ days after the herbicides application, water samples were collected to evaluate the physical chemical water parameters, herbicides concentration and zooplankton community. The water physical chemical parameters means were: dissolved oxygen $\left(3.5 \mathrm{mg} \mathrm{L}^{-1}\right)$, temperature $\left(20.1^{\circ} \mathrm{C}\right), \mathrm{pH}(6.0)$, total hardness $\left(18 \mathrm{mg} \mathrm{L}^{-1}\right.$ $\left.\mathrm{CaCO}_{3}\right)$ and total alkalinity $\left(9 \mathrm{mg} \mathrm{L}^{-1} \mathrm{CaCO}_{3}\right)$. The decreasing of herbicides persistence in water was: Clomazone $=$ Quinclorac $>$ Propanil > Metsulfuron-methyl with average of 31, 31, 10 and 7 days, respectively. The results indicated that the herbicides provoke little alteration in density of Rotifers and, Copepods (Adults and Nauplii). The Cladocers group density remained low for the whole experiment period.

Key words: agrochemicals, Crustacea, non-target organism, water quality, Southern Brazil, aquaculture ponds.

\section{INTRODUÇÃO}

O zooplâncton é constituído por importantes organismos do ecossistema aquático, que ocupam uma posição central da cadeia alimentar, já que transferem energia dos produtores primários para os organismos de níveis tróficos mais elevados, como os peixes. A estrutura de sua comunidade, biomassa e produção influenciam toda a estrutura do ciclo alimentar em ecossistema de água doce (MILLS \& FORNEY, 1988).

\footnotetext{
${ }^{\text {IC }}$ urso de Agronomia, Departamento de Defesa Fitossanitária, Universidade Federal de Santa Maria (UFSM), 97105-970, Santa Maria, RS, Brasil. Email: geovane_reimche@yahoo.com.br. Autor para correspondência.

IIDepartamento de Defesa Fitossanitária, UFSM, Santa Maria, RS, Brasil.

IIIUFSM, Santa Maria, RS, Brasil.

${ }^{\mathrm{IV}}$ Curso de Ciências Biológicas, Departamento de Ciências Naturais e Exatas, UFSM, Santa Maria, RS, Brasil.

vUniversidade Federal de Santa Catarina (UFSC), Florianópolis, SC, Brasil.

${ }^{\mathrm{VI}}$ Departamento de Fitotecnia, UFSM, Santa Maria, RS, Brasil.

${ }^{\mathrm{VII} D e p a r t a m e n t o ~ d e ~ Q u i ́ m i c a, ~ U F S M, ~ S a n t a ~ M a r i a, ~ R S, ~ B r a s i l . ~}$
} 
Entre muitas substâncias químicas tóxicas, os agroquímicos afetam o zooplâncton em nível individual, populacional e na comunidade (GOODRICH \& LEACH, 1990), constituindo-se em fonte de contaminação e, uma vez na água, espalham-se por todo o meio líquido, sendo difícil conter sua dispersão e ação tóxica sobre outros organismos.

Atualmente, os agroquímicos usados na lavoura arrozeira são bastante diversificados, muitos deles com níveis de toxicidade muito baixos e com degradação rápida no ambiente. Todavia, existem também aqueles produtos que oferecem altos riscos ambientais e que se encontram disponíveis no mercado, apesar de haver todo um processo oficial de liberação/ comercialização.

Nos Estados do Rio Grande do Sul (RS) e de Santa Catarina (SC), lavouras cultivadas com arroz irrigado são apontadas como grandes contaminantes ambientais, liberando no ambiente agroquímicos que podem chegar aos mananciais hídricos (MACHADO, 2003). Segundo KOLPIN et al. (1998) e HUBER et al. (2000), herbicidas persistentes e com grande mobilidade têm sido detectados em águas de superfície e subterrâneas, representando riscos para o ambiente, e prejudicando a qualidade da água. Inúmeros estudos indicam que estressores naturais podem alterar a sensibilidade da comunidade zooplanctônica a agroquímicos, fazendo com que espécies de zooplâncton, em ambiente natural, sejam mais sensíveis do que quando cultivadas em condições de laboratório (HANAZATO, 2001).

Clomazone, Quinclorac, Metsulfuron-methyl e Propanil são herbicidas recomendados para o controle de plantas daninhas em arroz irrigado no Sul do Brasil (SOSBAI, 2005). Na maioria das lavouras, as aplicações são seguidas de inundação da área e, em alguns casos, os herbicidas são aplicados diretamente na água de irrigação. Dependendo do manejo da água adotado e da precipitação pluvial após a aplicação, existe o risco de que resíduos desses produtos sejam carreados para fora da área (SUDO et al., 2002).

Embora se observe o uso corrente de herbicidas na lavoura arrozeira e haja possibilidade de contaminação de áreas adjacentes a esta, as pesquisas de monitoramento de herbicidas na água de irrigação são recentes no Brasil. Em vista disso, conduziu-se um experimento com o objetivo de determinar a persistência na água e a influência de Clomazone, Quinclorac, Metsulfuron-methyl e Propanil na comunidade zooplanctônica de água doce.

\section{MATERIAL E MÉTODOS}

O trabalho foi realizado durante 51 dias (31/ 03/2005 a 20/05/2005), em viveiros de aqüicultura localizados em área de várzea do Departamento de Fitotecnia da UFSM, em Santa Maria (RS). O delineamento experimental foi inteiramente casualizado com quatro repetições. Foram escavadas unidades experimentais (viveiros de aqüicultura) com capacidade de $22,5 \mathrm{~m}^{3}(5,0 \times 3,0 \times 1,5 \mathrm{~m})$, aplicando-se os herbicidas: Clomazone [2-[(2-clorobenzil)]-4,4-dimetil-1,2oxazolidin-3-ona] $\left(0,5 \mathrm{mg} \mathrm{L}^{-1}\right)$, Quinclorac [3,7dicloroquinolina-8-ácido carboxílico] $\left(0,375 \mathrm{mg} \mathrm{L}^{-1}\right)$, Metsulfuron-methyl (metil2-[(4-metoxi-6-metil-1,3,5triazina-2-il) a mino ] carbonil] a mino ] sulfonil]benzoatosulfoniluréia) $\left(0,002 \mathrm{mg} \mathrm{L}^{-1}\right)$ e Propanil [2-metil-3,4-dicloro acetanilida] $\left(3,6 \mathrm{mg} \mathrm{L}^{-1}\right)$, totalizando 4 tratamentos mais o tratamento controle (sem herbicida).

As coletas de água para análise foram realizadas no $1^{\circ}, 3^{\circ}, 7^{\circ}, 10^{\circ}, 18^{\circ}, 31^{\circ}$ e $51^{\circ} \stackrel{\text { dia após a }}{ }$ aplicação dos herbicidas e as amostras armazenadas em frascos de vidro âmbar de 1 litro. As amostras foram analisadas no Laboratório de Análises de Resíduos de Pesticidas (LARP) da UFSM, segundo descrito por ZANELLA et al. (2000), empregando-se cromatografia líquida de alta performance com detecção no ultravioleta (HPLC-UV). A persistência dos herbicidas em água foi definida como o período em dias entre a aplicação dos herbicidas e a última coleta com concentração quantificável.

Os parâmetros físico-químicos da água determinados foram: $\mathrm{pH}$ ( $\mathrm{pHmetro}$ Schott Handylab 1), temperatura e oxigênio dissolvido (Oxímetro Oakton) e alcalinidade e dureza total (APHA, 1992).

O zooplâncton foi amostrado no $1^{\circ}$, $2^{\circ}$, $3^{\circ}$, 7o, $10^{\circ}, 18^{\circ}, 31^{\circ}$ e $51^{\circ}$ dia após a aplicação dos herbicidas, utilizando-se uma rede coletora de plâncton $(25 \mu \mathrm{m})$ no horário compreendido entre $4 \mathrm{~h} 30 \mathrm{~min}$ e $6 \mathrm{~h} 30 \mathrm{~min}$, sendo as amostras fixadas em formol (4\%). Posteriormente, com o auxílio de uma pipeta volumétrica, retiraram-se de cada amostra subamostras que foram separadas em placa de Bogorov para análise quali-quantitativa dos grupos zooplanctônicos, empregando-se o uso de microscópio esteroscópio.

Os resultados da densidade de cada grupo do zooplâncton (Cladocera, Copepoda e Rotifera) foram submetidos à análise de variância (ANOVA) e as médias foram comparadas pelo teste de Tukey $(\mathrm{P}<0,05)$. Para verificar as associações existentes entre os parâmetros físico-químicos da água (grupo I) e a comunidade zooplanctônica (grupo II), procedeu-se à análise de correlação canônica (CRUZ \& REGAZZI, 1994), utilizando-se para isso a covariância entre os dois grupos.

\section{RESULTADOS E DISCUSSÃO}

As concentrações mais altas dos herbicidas na água ocorreram nos primeiros dias após a aplicação, 
decrescendo com o tempo de amostragem, com variação entre os herbicidas analisados (Tabela 1), que demonstram consistência e concordância com dados já reportados (CAPRI et al., 1999; MACHADO et al., 2003). Os herbicidas Clomazone e Quinclorac foram mais persistentes na água, sendo detectados até 31 dias após a aplicação, corroborando os resultados obtidos por outros pesquisadores (CUMMING et al., 2002). No Brasil, foi detectado Clomazone na água de irrigação até 32 dias após à aplicação, em concentrações na ordem de $0,6 \mathrm{mg} \mathrm{L}^{-1}$ (NOLDIN et al., 1997), sendo também o herbicida mais freqüentemente encontrado em águas de rios (MARCHEZAN et al., 2003).

A concentração de Quinclorac manteve-se alta até o $7^{\circ}$ dia $\left(102 \mathrm{mg} \mathrm{L}^{-1}\right)$, sendo detectada até o $31^{\circ}$ dia (Tabela 1). Em condições de campo, Quinclorac persistiu até 31 dias, ao contrário dos resultados encontrados nos testes de laboratório (CROSBY, 2003). O Propanil apresentou a menor persistência (Tabela 1). A redução da concentração do produto de 3.600 para $10 \mathrm{mg} \mathrm{L}^{-1}$, no 7 o dia, deve-se, provavelmente a sua degradação rápida em meio aquoso.

Os parâmetros físico-químicos da água (Tabela 2) demonstraram que os níveis de oxigênio dissolvido variaram entre 2,4 a 4,6 $\mathrm{mg} \mathrm{L}^{-1}$, considerados baixos para organismos aquáticos. A temperatura da água variou de 16,4 a $23,9^{\circ} \mathrm{C}$ e o $\mathrm{pH}$ de 5,5 a 6,1 . Na última amostragem, o aumento do $\mathrm{pH}$ deve-se provavelmente à redução na fixação de $\mathrm{CO}_{2}$ da água pelo fitoplâncton, provocado pela menor temperatura. Já a dureza total variou de 14 a $20 \mathrm{mg} \mathrm{L}^{-1} \mathrm{de} \mathrm{CaCO}_{3}$ e a alcalinidade total entre 5 e $14 \mathrm{mg} \mathrm{L}^{-1}$ de $\mathrm{CaCO}_{3}$.

Os pares canônicos foram significativos apenas para a primeira ordem $(\mathrm{r}=0,74)(\mathrm{P}<0,004)$ (Tabela 3 ). Os grupos considerados (parâmetros físicoquímicos e grupos zooplanctônicos) não são independentes e as associações intergrupos foram estabelecidas da seguinte forma: quando a água está com temperatura mais elevada e com menores valores de $\mathrm{pH}$, há uma tendência na redução da densidade do grupo Nauplio. Estes resultados estão de acordo com RIETZLER (1995), que, estudando a dinâmica de populações do grupo Copepoda na Represa do Lobo (Itirapina, Brotas - São Paulo), observou alta mortalidade de Nauplio com a elevação da temperatura da água.

Para Cladocera (Tabela 4), não houve diferença significativa entre os tratamentos até o $7^{\circ}$ dia. Entretanto, a partir do $10^{\circ}$ dia, em geral, o tratamento com Quinclorac apresentou aumento na densidade $(\mathrm{P}<0,05)$ em relação ao controle. Já para Clomazone, a partir do $18^{\circ}$ dia houve uma tendência ao aumento de organismos deste grupo. Na comparação entre os dias amostrados, Metsulfuron-methyl e Propanil apresentaram baixa densidade de organismos, assim como no tratamento controle. Dessa forma, não é possível afirmar que os herbicidas em estudo afetaram ou não o grupo Cladocera, pois este se manteve em baixas densidades durante maior parte do período amostrado. A baixa população de Cladocera pode ser atribuída ao fato deste grupo gerar formas de estágios latentes duradouros (POLLARD et al., 2003).

Quanto à Rotifera (Tabela 4), não se verificou diferença significativa entre os tratamentos nas três primeiras amostragens. Por outro lado, com a aplicação de Propanil e Metsulfuron-methyl, houve redução da densidade na amostragem do $7^{\circ}$ dia em relação ao controle. Para Clomazone e Quinclorac, no $7^{\circ}$ e no $10^{\circ}$ dia, não foram observadas diferenças em relação ao controle. Já no $18^{\circ}$ dia, ocorreu um aumento significativo na densidade deste grupo, com posterior redução a partir do $31^{\circ}$ dia.

Comparando os dias amostrais, houve recuperação de Rotifera a partir do 7o dia para os herbicidas Clomazone e Quinclorac, assim como no controle. Propanil e Metsulfuron-methyl, mantiveram-

Tabela 1 - Concentração e persistência dos herbicidas aplicados nos viveiros de aqüicultura.

\begin{tabular}{|c|c|c|c|c|c|c|c|c|}
\hline \multirow{2}{*}{ Herbicida } & \multicolumn{7}{|c|}{ Concentração $\left(\mu \mathrm{g} \mathrm{L}^{-1}\right)^{2}$} & \multirow{2}{*}{$\begin{array}{l}\text { Persistênci } \\
\qquad{\text { (dias })^{4}}^{-}\end{array}$} \\
\hline & Inicial $^{1}$ & $1^{\circ}$ & $3^{-}$ & $7^{\underline{0}}$ & $10^{\circ}$ & $18^{\mathrm{o}}$ & Final $^{3}$ & \\
\hline Clomazone & 500 & 376 & 322 & 127 & 70,8 & 47 & 5,6 & 31 \\
\hline Quinclorac & 375 & 204 & 121 & 102 & 60,7 & 13 & 6,4 & 31 \\
\hline Metsulfuron-methyl & 2 & 1,35 & 1,27 & 1,0 & $\mathrm{nd}^{5}$ & nd & 1,0 & 7 \\
\hline Propanil & 3.600 & 1.644 & 317 & 10 & 0,5 & nd & 0,5 & 10 \\
\hline
\end{tabular}

${ }^{1}$ Concentração teórica, em $\mu \mathrm{g} \mathrm{L}{ }^{-1}$, na água.

${ }^{2} \mathrm{O}$ limite de quantificação do método analítico após a etapa de pré-concentração foi de $0,1 \mu \mathrm{g} \mathrm{L^{-1 }}$. para Clomazone e Propanil, e de $0,5 \mu \mathrm{g} \mathrm{L}^{-1}$ para o Quinclorac e o Metsulfuron-methyl.

${ }^{3}$ Concentração encontrada na última coleta que ainda apresentou resíduo quantificável de herbicida.

${ }^{4}$ Data da última coleta em que foi quantificado o herbicida.

${ }^{5}$ Não detectável. 
Tabela 2 - Média dos parâmetros físico-químicos da água nos viveiros de aqüicultura durante o experimento.

\begin{tabular}{|c|c|c|c|c|c|}
\hline \multirow{2}{*}{$\begin{array}{l}\text { Amostragem } \\
\text { (dia) }\end{array}$} & \multicolumn{5}{|c|}{ Parâmetros avaliados ${ }^{2}$} \\
\hline & $\mathrm{OD}^{1}$ & $\mathrm{~T}\left({ }^{\circ} \mathrm{C}\right)$ & $\mathrm{pH}$ & Dureza total $^{1}$ & Alcalinidade total $^{1}$ \\
\hline $1^{\circ} \underline{ }$ & 2,7 & 21,5 & 5,9 & 20 & 8 \\
\hline $2^{\circ}$ & 4,1 & 23,9 & 6,1 & 20 & 8 \\
\hline $3^{\mathrm{o}}$ & 3,8 & 21,1 & 5,5 & 18 & 7 \\
\hline $7^{\mathrm{o}}$ & 3,4 & 20,7 & 6,0 & 19 & 8 \\
\hline $10^{\mathrm{o}}$ & 2,4 & 21,6 & 5,8 & 14 & 7 \\
\hline $18^{\circ}$ & 4,6 & 18,4 & 6,1 & 14 & 5 \\
\hline $31^{\circ}$ & 4,3 & 16,4 & 6,0 & 18 & 14 \\
\hline $51^{\mathrm{o}}$ & 2,9 & 17,1 & 6,5 & 19 & 11 \\
\hline Média & 3,5 & 20,1 & 6,0 & 18 & 9,0 \\
\hline
\end{tabular}

${ }^{1}$ Oxigênio dissolvido $\left(\mathrm{mg} \mathrm{L}^{-1}\right)$, dureza total $\left(\mathrm{mg} \mathrm{L}^{-1} \mathrm{de} \mathrm{CaCO}_{3}\right)$, alcalinidade total $\left(\mathrm{mg} \mathrm{L}^{-1}\right.$ de $\left.\mathrm{CaCO}_{3}\right)$.

${ }^{2}$ Média de valores entre os tratamentos.

se, de uma forma geral, com pequenas alterações na densidade, ao longo dos dias.

Os resultados do presente experimento mostram que Rotifera foi menos sensível a agroquímicos, assim como verificado por HAVENS \& HANAZATO (1993). Concordando com isto, NEVES et al. (2003) afirmam que o grupo Rotifera, por ser constituído de organismos pequenos e apresentar ciclo curto de vida, possui ampla tolerância à variabilidade de fatores ambientais.

Quanto à Copepoda Adulto (Tabela 4), podese verificar que até o $31^{\circ}$ dia não foram observadas diferenças dos tratamentos com herbicidas em relação ao controle. Já no $51^{\circ}$ dia, nos tratamentos com
Clomazone e Propanil, ocorreram densidades significativamente maiores quando comparadas ao controle. Analisando o comportamento do grupo entre as amostragens, verificou-se que para Propanil ocorreu um aumento significativo de organismos no $2^{\circ}$ dia e uma queda acentuada a partir do $3 \stackrel{\circ}{\circ}$ dia, permanecendo assim até a amostragem no $31^{\circ}$ dia. Não houve diferenças na densidade deste grupo para Quinclorac e Metsulfuron-methyl. O tratamento Clomazone seguiu os mesmos padrões de densidade de organismos do controle.

Copepoda Adulto, comparado aos demais, foi o grupo que apresentou as mais altas densidades de organismos nos três primeiros dias iniciais de

Tabela 3 - Média, desvio padrão e correlações canônicas entre os parâmetros físico-químicos da água (Grupo I) e os organismos zooplanctônicos (Grupo II).

\begin{tabular}{llll}
\hline & & \multicolumn{2}{c}{ Pares canônicos } \\
Grupos I e II & $\mathrm{x} \pm \mathrm{DS}^{1}$ & $1^{\mathrm{o}}$ & $2^{\underline{0}}$ \\
\cline { 3 - 4 } Oxigênio $\left(\mathrm{mg} \mathrm{L}^{-1}\right)$ & $3,53 \pm 0,87$ & 0,1901 & 0,3912 \\
Temperatura $\left({ }^{\circ} \mathrm{C}\right)$ & $20,12 \pm 2,45$ & 0,7241 & $-0,6679$ \\
$\mathrm{pH}$ & $5,97 \pm 0,36$ & $-0,6485$ & $-0,4554$ \\
Dureza $\left(\mathrm{mg} \mathrm{L}^{-1} \mathrm{CaCO}_{3}\right)$ & $17,65 \pm 5,89$ & $-0,1113$ & 0,1568 \\
Alcalinidade $\left(\mathrm{mg} \mathrm{L}^{-1} \mathrm{CaCO}_{3}\right)$ & $8,49 \pm 3,76$ & 0,0801 & $-0,4108$ \\
& & & 0,1536 \\
Cladocera $^{2}$ & $0,53 \pm 0,77$ & $-0,2433$ & $-0,6640$ \\
Copepoda Adulto $^{2}$ & $4,01 \pm 3,29$ & 0,3177 & $-0,1604$ \\
Copepoda Nauplio $^{2}$ & $2,10 \pm 2,41$ & $-0,9810$ & 0,0959 \\
Rotifera $^{1}$ & $3,00 \pm 3,06$ & $-0,2613$ & 0,5137 \\
r $(\rho)^{3}$ & - & 0,7488 & 0,5451 \\
Significância $(\alpha)$ & - & 0,0041 & 11,88 \\
$x^{2}$ & - & 40,68 & \\
\hline
\end{tabular}

\footnotetext{
${ }^{1} \mathrm{x} \pm$ DS. $=$ média \pm desvio padrão.

${ }^{2}$ Organismos $\mathrm{L}^{-1}$.

${ }^{3}$ Coeficiente de correlação.
}

Ciência Rural, v.38, n.1, jan-fev, 2008. 
Persistência na água e influência de herbicidas utilizados na lavoura arrozeira sobre a comunidade zooplanctônica...

Tabela 4 - Densidade populacional (organismos $\mathrm{L}^{-1}$ ) de Cladocera, Rotifera, Copepoda (Adulto e Nauplio) durante o período de amostragem.

\begin{tabular}{|c|c|c|c|c|c|c|c|c|}
\hline \multirow[b]{2}{*}{ Tratamentos } & \multicolumn{8}{|c|}{ Amostragens (dias) } \\
\hline & $1^{\mathrm{o}}$ & $2^{\mathrm{o}}$ & $3^{-}$ & $7^{\underline{0}}$ & $10^{\mathrm{o}}$ & $18^{\mathrm{o}}$ & $31^{\mathrm{o}}$ & $51^{\circ}$ \\
\hline & \multicolumn{8}{|c|}{ Cladocera } \\
\hline Controle & ${ }^{\mathrm{NS}} 0^{\mathrm{ns}}$ & $0^{\mathrm{ns}}$ & $0^{\mathrm{ns}}$ & $1^{\mathrm{ns}}$ & $0 \mathrm{~b}$ & $0^{\text {ns }}$ & $0 \mathrm{bc}$ & $0 \mathrm{~b}$ \\
\hline Clomazone & B 0 & B 0 & B 0 & В 0 & B $0 \mathrm{~b}$ & $\mathrm{AB} 1$ & A $2 \mathrm{ab}$ & $\mathrm{AB} 1 \mathrm{ab}$ \\
\hline Quinclorac & $\mathrm{CO}$ & $\mathrm{C} 0$ & $\mathrm{Co}$ & $\mathrm{BC} 0$ & $\mathrm{AB} 2 \mathrm{a}$ & $\mathrm{ABC} 1$ & A 3 a & A 3 a \\
\hline Metsulfuron & ${ }^{\mathrm{NS}} 0$ & 0 & 0 & 0 & $0 \mathrm{~b}$ & 0 & $0 \mathrm{c}$ & $1 \mathrm{~b}$ \\
\hline Propanil & ${ }^{\mathrm{NS}} 0$ & 0 & 0 & 0 & $0 \mathrm{~b}$ & 0 & $1 \mathrm{bc}$ & $0 \mathrm{~b}$ \\
\hline \multirow[t]{2}{*}{ Média } & 0 & 0 & 0 & 0 & 0 & 0 & 1 & 1 \\
\hline & \multicolumn{8}{|c|}{ Rotifera } \\
\hline Controle & $\mathrm{C} 2^{\mathrm{ns}}$ & $\mathrm{BC} 3^{\mathrm{ns}}$ & $\mathrm{BC} 3^{\mathrm{ns}}$ & A $12 \mathrm{a}$ & $\mathrm{ABC} 5 \mathrm{a}$ & $\mathrm{C} 1 \mathrm{~b}$ & $\mathrm{AB} 8 \mathrm{ab}$ & $\mathrm{C} 2^{\mathrm{ns}}$ \\
\hline Clomazone & $\mathrm{C} 1$ & $\mathrm{BC} 1$ & $\mathrm{BC} 2$ & $\mathrm{AB} 5 \mathrm{ab}$ & $\mathrm{ABC} 3 \mathrm{ab}$ & A $11 \mathrm{a}$ & $\mathrm{BC} 2 \mathrm{c}$ & $\mathrm{BC} 1$ \\
\hline Quinclorac & $\mathrm{BC} 2$ & $\mathrm{BC} 1$ & $\mathrm{C} 1$ & $\mathrm{AB} 6 \mathrm{a}$ & $\mathrm{AB} 5 \mathrm{a}$ & A $8 \mathrm{a}$ & $\mathrm{ABC} 3 \mathrm{bc}$ & $\mathrm{ABC} 4$ \\
\hline Metsulfuron & B 1 & B 1 & B 0 & B $1 b c$ & B 2ab & B $2 b$ & A $10 \mathrm{a}$ & B 1 \\
\hline Propanil & $\mathrm{BC} 0$ & $\mathrm{ABC} 1$ & $\mathrm{C} 0$ & $\mathrm{C} \mathrm{0c}$ & $\mathrm{ABC} 1 \mathrm{~b}$ & A $4 \mathrm{ab}$ & $\mathrm{AB} 4 \mathrm{abc}$ & $\mathrm{ABC} 3$ \\
\hline \multirow[t]{2}{*}{ Média } & 1 & 1 & 1 & 5 & 3 & 5 & 5 & 2 \\
\hline & \multicolumn{8}{|c|}{ Copepoda Adulto } \\
\hline Controle & $\mathrm{AB} 5^{\mathrm{ns}}$ & A $8 \mathrm{ab}$ & $\mathrm{AB} 3^{\text {ns }}$ & $\mathrm{AB} 3^{\text {ns }}$ & $\mathrm{B} 1^{\mathrm{ns}}$ & $\mathrm{B} 1^{\mathrm{ns}}$ & $\mathrm{AB} 3^{\mathrm{ns}}$ & $\mathrm{AB} 2 \mathrm{~b}$ \\
\hline Clomazone & $\mathrm{AB} 8$ & $\mathrm{AB} 5 \mathrm{~b}$ & $\mathrm{AB} 4$ & B 2 & B 2 & B 3 & B 3 & A $11 \mathrm{a}$ \\
\hline Quinclorac & ${ }^{\mathrm{NS}} 2$ & $2 b$ & 2 & 2 & 2 & 1 & 2 & $5 \mathrm{ab}$ \\
\hline Metsulfuron & ${ }^{\mathrm{NS}} 4$ & $5 b$ & 6 & 3 & 3 & 1 & 1 & $5 \mathrm{ab}$ \\
\hline Propanil & $\mathrm{BC} 6$ & A $18 \mathrm{a}$ & $\mathrm{BC} 6$ & BC 3 & C 1 & $\mathrm{C} 1$ & $\mathrm{C} 2$ & $\mathrm{AB} 9 \mathrm{a}$ \\
\hline \multirow[t]{2}{*}{ Média } & 5 & 8 & 4 & 3 & 2 & 1 & 2 & 7 \\
\hline & \multicolumn{8}{|c|}{ Copepoda Nauplio } \\
\hline Controle & ${ }^{\mathrm{NS}} 1^{\mathrm{ns}}$ & $3^{\text {ns }}$ & $1^{\text {ns }}$ & $2^{\mathrm{ns}}$ & $1^{\mathrm{ns}}$ & $1^{\mathrm{ns}}$ & $3^{\mathrm{ns}}$ & $1 \mathrm{c}$ \\
\hline Clomazone & B 0 & B 1 & В 0 & B 3 & B 1 & B 3 & B 3 & A $13 a$ \\
\hline Quinclorac & B 1 & B 2 & B 1 & B 1 & B 0 & B 2 & AB 3 & A $9 a b$ \\
\hline Metsulfuron & ${ }^{\mathrm{NS}} 2$ & 1 & 1 & 3 & 1 & 1 & 1 & $5 b c$ \\
\hline Propanil & $\mathrm{AB} 2$ & $\mathrm{AB} 2$ & B 1 & B 1 & B 1 & В 0 & B 1 & A $7 \mathrm{ab}$ \\
\hline Média & 1 & 2 & 1 & 2 & 1 & 2 & 2 & 7 \\
\hline
\end{tabular}

*Na linha, médias não antecedidas da mesma letra maiúscula e, na coluna, médias não seguidas da mesma letra minúscula diferem entre si pelo teste de Tukey em nível de $5 \%$ de probabilidade de erro.

${ }_{\text {ns/NS }}$ Teste F não-significativo em nível de $5 \%$ de probabilidade.

amostragem, corroborando com CÁCERES \& SOLUK (2002), que afirmam que Copepoda apresenta habilidade de colonizar mais rapidamente o novo habitat quando comparado com Cladocera. Estudos realizados por BONACINA \& PASTERIA (2001) mostraram que Copepoda e algumas espécies de Rotifera foram pioneiras na colonização de um lago acidificado estéril na Itália.

Para Copepoda Nauplio, até o $31^{\circ}$ dia de coleta, sua densidade manteve-se constante, ocorrendo apenas no 51을 dia um aumento significativo $(\mathrm{P}<0,05)$ nas densidades nos tratamentos com Clomazone, Quinclorac e Propanil em relação ao controle. Já no decorrer das coletas, as densidades de organismos deste grupo mostraram-se inalteráveis, verificando-se pouca influência dos tratamentos para com este grupo. Este fato foi confirmado por LAMPERT et al. (1989), que observaram maior grau de tolerância de Copepoda Adulto e Nauplio, em ambiente contaminado pelo herbicida atrazine.

A reduzida densidade de Rotifera no início e final das coletas pode estar relacionada à maior densidade de Copepoda, responsável pela predação de Rotifera. Estudos de GILBERT (1988) ressaltam que Rotifera é mais abundante quando Cladocera encontrase em menor número de organismos. Entre os grupos, a redução na população não pode ser atribuída exclusivamente aos herbicidas aplicados, pois outros 
fatores de ordem biológica podem interagir com os agrotóxicos, tendo conseqüências na população destes grupos (JAK et al., 1996). Segundo GAGNETEN (2002), os herbicidas podem provocar redução na densidade do zooplâncton, em especial na de crustáceos herbívoros (Cladocera e Copepoda), por determinar a diminuição dos recursos alimentares e trocas na estrutura da comunidade algal e, associado a isto, podem apresentar efeitos tóxicos.

Os resultados apresentados permitem, ainda, uma aplicação ampla, podendo servir de subsídios para programas de monitoramento de bacias hidrográficas que recebem aporte de água drenada de lavouras de arroz irrigado, no sentido de adoção de procedimentos que evitem ou minimizem os riscos de contaminação ambiental. Entre esses procedimentos estão a seleção e aplicação de herbicidas que, preferencialmente, apresentem degradação rápida. Além dos herbicidas, outros agroquímicos devem ser testados, uma vez que esses compostos, nas suas diversas transformações, podem gerar também compostos nocivos ao meio ambiente.

\section{CONCLUÇÕES}

Pelos resultados obtidos com os herbicidas testados, depreende-se que a água deva ser mantida na lavoura por cerca de 31 dias após a aplicação do produto, como medida inicial de segurança.

Os herbicidas Clomazone, Quinclorac, Metsulfuron-methyl e Propanil provocaram poucas alterações na densidade da comunidade zooplanctônica para os grupos Rotifera e Copepoda (Adulto e Nauplio). A densidade do grupo Cladocera permaneceu baixa ao longo do experimento.

\section{AGRADECIMENTOS}

Os autores agradecem à UFSM e ao Conselho Nacional de Desenvolvimento Científico e Tecnológico (CNPq), pelo suporte financeiro e pela bolsa de iniciação científica para o estudante G. B. Reimche.

\section{REFERÊNCIAS}

APHA (American Public Health Association). Standard methods for examination of water and wastewater. 18.ed. Sprinfield: Bru-El Graphic, 1992. 1155p.

BONACINA C.; PASTERIA, A. Zooplankton of Lake Orta after liming: an eleven year study. Journal of Limnology, Verbania, v.60, p.101-109, 2001.

CÁCERES, C.E.; SOLUK, D.A. Blowing in the wind: a field test of overland dispersal and colonization by aquatic invertebrates. Oecologia, Heidelberg, v.131, p.402-408, 2002.
CAPRI, E. et al. Ground and surface water bodies contamination by pesticides use in paddy field. In: Environmental risk parameters for use of plant protection products in rice. Piacenza: Tipolitografia, 1999. p.48-71.

CROSBY, D.R. Environmental fate of pesticides-87. Capturado em 10 mar. 2003. Online. Disponível na internet http://www.syix.com/rrb/87rpt/Enviro.htm.

CRUZ, C.D.; REGAZZI, A.J. Modelos biométricos aplicados ao melhoramento genético. Viçosa: UFV, 1994. 390p.

CUMMING, J.P. et al. Clomazone dissipation in four Tasmanian topsoils. Weed Science, Lawrence, v.50, p.405-409, 2002.

GAGNETEN, A.M. Efectos del herbicida paraquat sobre el zooplancton. Iheringia, Porto Alegre, v.92, p.47-56, 2002.

GILBERT, J.J. Suppression of Rotifer population by Daphnia: a review of the evidence, mechanisms, and the effect on the zooplankton community structure. Limnology and Oceanography, Waco, v.33 p.1286-1303, 1988.

GOODRICH, M.S.; LEACH, J.J. A behavioral screening assay for Daphnia magna: a method to assess the effects of xenobiotics on spatial orientation. Environmental Toxicology and Chemistry, Houston, v.9, p.21-30, 1990.

HANAZATO, T. Pesticide effects on freshwater zooplankton: an ecological perspective. Environmental Pollution, Amherst, v.112, p.1-10, 2001.

HAVENS, K.E.; HANAZATO, T. Zooplankton community responses to chemical stressors: a comparison of results from acidification and pesticide contamination research. Environmental Pollution, Amherst, v.82, p.277-288, 1993.

HUBER, A. et al. Pollution of surface waters with pesticides in Germany: modeling non-point source inputs. Agriculture, Ecosystems and Environment, Charlottetown, v.80, p.191204,2000 .

JAK, R.G. et al. Evaluation of laboratory derived toxic effect concentration of a mixture of metals by testing fresh water plankton communities in enclosures. Water Research, Elmsford, v.30, p.1215-1227, 1996.

KOLPIN, D.W. et al. The environmental occurrence of herbicides: The Importance of degradates in ground water. Archieves of Environmental Contamination and Toxicology, New York, v.35, p.385-390, 1998.

LAMPERT W. et al. Herbicides effect on planktonic systems of different complexity. Hydrobiology, Brussels, v.188/189, p.415-424, 1989.

MACHADO, S.L. de O. et al. Persistência de herbicidas na água de irrigação no arroz irrigado. In: CONGRESSO BRASILEIRO DE ARROZ IRRIGADO, 3; REUNIÃO DA CULTURA DO ARROZ IRRRIGADO, 25., Balneário Camboriú, 2003. Anais... Itajaí: EPAGRI, 2003. p.692-694.

MACHADO, S.L. de O. Sistemas de estabelecimento do arroz irrigado, consumo de água, perdas de nutrientes, persistência de herbicidas na água e efeito no jundiá. 
2003. 147f. Tese (Doutorado em Agronomia) - Universidade Federal de Santa Maria.

MARCHEZAN, E. et al. Dispersão dos herbicidas clomazone, quinclorac e propanil nas águas da Bacia Hidrográfica dos rios Vacacaí e Vacacaí-Mirim, no período de cultivo do arroz irrigado. In: CONGRESSO BRASILEIRO DE ARROZ IRRIGADO, 3.; REUNIÃO DA CULTURA DO ARROZ IRRRIGADO, 25. Balneário Camboriú, 2003. Anais... Itajaí: EPAGRI, 2003. p.689-691

MILLS, E.L.; FORNEY, J.L. Trophic dynamics and development of freshwater pelagic food webs. In: CARPENTER, S.R. Complex Interactions in Lake Communities. New York: Springer-Verlag, 1998. p.11-30.

NOLDIN, J.A. et al. Persistência do herbicida clomazone em arroz irrigado em sistema pré-germinado. In: REUNIÃO DA CULTURA DO ARROZ IRRIGADO, 22., 1997. Balneário Camboriú. Anais... Itajaí: EPAGRI, 1997. p.363-364.

NEVES, I.F. et al. Zooplankton community structure of two marginal lakes of the river Cuiabá (Mato Grosso, Brazil) with analysis of Rotifera and Cladocera diversity. Brazilian Journal Biology, São Carlos, v.63, p.329-343, 2003.
POLLARD, H.G. et al. Reconstruction of centuries-old Daphnia communities in a lake recovering from acidification and metal contamination. Ambio, Stockholm, v.32, p.214-218, 2003.

RIETZLER, A.C. et al. Estudo da dinâmica de populações de Copepoda Calonoida da Represa do Lobo (Broa). 1995. 68f. Dissertação (Mestrado em Ciências da Engenharia Ambiental) - Escola de Engenharia de São Carlos, Universidade de São Paulo (USP).

SOCIEDADE SUL-BRASILEIRA DE ARROZ IRRIGADO (SOSBAI). Arroz irrigado: recomendações técnicas da pesquisa para o Sul do Brasil. Santa Maria, RS: SOSBAI, 2005. 159p.

SUDO, M. et al. Concentration and loading pesticides residues in Lake Biwa Basin (Japan). Water Research, Elmsford, v.36, p.315-329, 2002.

ZANELLA R. et al. Development and validation of a highperformace liquid chromatographic procedure for the determination of clomazone residues in surface water. Journal of Chromatography A, Amsterdam, v.904, p.257-262. 